\title{
Agua y Estado como ejes estructurantes de los procesos de transformación del hábitat rural en el árido de Mendoza (Argentina)
}

\author{
Water and Government as structuring axes of the \\ processes of habitat transformation in the arid of \\ Mendoza (Argentina)
}

\author{
Matías J. Esteves ${ }^{1}$ V Virginia Miranda Gassull|2
}

\begin{abstract}
RESUMEN
Se considera al hábitat rural de tierras secas no irrigadas de Mendoza (Argentina) con un patrón disperso de ocupación del suelo y vinculado exclusivamente a la cría de ganado caprino. Pero las transformaciones territoriales vinculadas a la escasez de agua superficial sumado a acciones estatales en materia de servicios e infraestructura han incidido en la configuración del hábitat, detectándose actualmente población agrupada que conforma pequeños poblados. Se utilizan principalmente métodos cualitativos -observación directa y entrevistas en profundidad- aplicados en dos casos de estudio. Se indaga en los aspectos institucionales y ecológicos que inciden en la configuración-transformación del hábitat en relación con su trayectoria histórica. Los resultados muestran que los poblados poseen características propias y diferentes al hábitat disperso, donde su consideración permitiría enriquecer la enunciación de políticas en pos de visibilizar las dinámicas presentes en el territorio y la heterogeneidad en la configuración del hábitat.

Palabras claves: Hábitat rural, Zonas áridas, Políticas estatales, Desertificación.
\end{abstract}

\begin{abstract}
Its consider that the rural habitat of the not irrigated dry land of Mendoza (Argentina) with a dispersed pattern of land occupation and linked mainly to the breeding of goats. But the territorial transformation linked with scarcity of superficial water in addition to the public policies in services and infrastructure have influences the habitat configuration, detecting diverse villages. From the use of qualitative methods, two villages are characterized as case studies. The institutional and ecological aspects that affect its configuration-transformation are investigated in relation to its historical trajectory. The results show that these villages have their own characteristics and that they are different from the dispersed habitat, where their consideration would enrich the enunciation of public policies in order to visualize the dynamics present in the territory and the heterogeneity of its configuration.
\end{abstract}

Key Words: Rural habitat, Dry Lands, Government politics, Desertification. 


\section{Introducción}

La dinámica de los asentamientos humanos ha estado directamente relacionada con el descubrimiento y apropiación de los bienes de la naturaleza y a los hechos socioeconómicos proyectados en el tiempo (Quiroga Martínez, 2007). En el abordaje de las tierras secas de clima árido destaca que el agua se convierte en el elemento organizador del territorio (de inclusión o exclusión), tanto por su disponibilidad como por su uso (Secretaría de Ambiente y Ordenamiento Territorial, 2017; Montaña, 2007). Dentro de las zonas rurales de tierras secas, la relación del hábitat humano con la naturaleza es más evidente ya que el tipo y envergadura de las actividades productivas se vincula directamente con la disponibilidad de bienes naturales, principalmente agua y suelo cultivable. Se coincide con A. Rapoport (2003) cuando sostiene que el hábitat tiene que ser aprehendido como un sistema de lugares donde interviene la combinación de factores sociales, económicos y ecológicos que permiten un acercamiento a la complejidad de los territorios rurales. En efecto, el hábitat rural no sólo implica el estudio de la vivienda, sino que también involucra a las actividades productivas y a las características del ecosistema, además de las relaciones sociales que adquieren gran protagonismo (Sánchez Quintanar y Jiménez Rojas, 2010; Castro y Reboratti, 2008).

En la provincia de Mendoza se encuentran, por un lado, las tierras secas irrigadas, es decir los territorios con acceso al agua superficial y por ende al riego y con posibilidad de cultivo -oasis- donde se asienta la mayor densidad poblacional. Por otro lado, las tierras secas no irrigadas que poseen escasa presencia de agua superficial, baja densidad poblacional y actividades productivas de subsistencia. En la literatura en general existe una tendencia a considerar que las tierras secas no irrigadas de Mendoza se caracterizan por la presencia de asentamientos humanos dispersos (Secretaría de Ambiente y Ordenamiento Territorial, 2017; Ministerio del interior, 2014; Abraham et al., 2014; Montaña, 2013). Esta consideración del territorio como homogéneo soslaya las riquezas que presentan las diversas formas de habitar e incide de forma negativa en la formulación de políticas acordes a la realidad. Ciertamente, en estos textos se omite la existencia de población agrupada que conforma pequeños poblados y que poseen características propias y diferentes a los asentamientos dispersos. Las tierras secas no irrigadas de Mendoza abarcan el $97 \%$ de la superficie provincial (Gudiño, 2017) y por ello, resulta relevante analizar las formas de configuración del hábitat. Actualmente se observa una tendencia clara hacia el agrupamiento de población, donde influye la disponibilidad de agua, la presencia de infraestructura y servicios básicos y mejores condiciones de accesibilidad. Estos poblados se caracterizan por la presencia de hasta 50 viviendas emplazadas en un radio de $350 \mathrm{~m}$ y por ello se habla de un patrón agrupado, mientras que los asentamientos dispersos se localizan entre sí a una distancia que oscila entre los $2 \mathrm{Km}$ a $10 \mathrm{Km}$ de distancia. A su vez, esta forma agrupada de habitar el territorio implica cambios en las actividades productivas que tradicionalmente realizan los habitantes. Por ello, el objetivo del trabajo es caracterizar a los poblados de la zona no irrigada e indagar en los aspectos institucionales y ecológicos que inciden en su configuración-transformación en relación con su trayectoria histórica.

Se trabaja en el noreste de la provincia, en la parte baja de la cuenca del río Mendoza. Se trata de un territorio habitado por comunidades indígenas Huarpes y caracterizado históricamente por su ocupación dispersa, aunque desde mediados del siglo XX se comienza a registrar una tendencia a la organización agrupada en poblados. Al interior del área, se han seleccionado como casos 
de estudio a dos distritos del departamento de Lavalle: Lagunas del Rosario y La Asunción. La selección de estos casos se justifica en que se trata de los dos distritos no irrigados con mayor población que atraviesa el río Mendoza y que presenta transformaciones vinculadas con la merma en la disponibilidad de agua superficial y fuertes procesos de desertificación. Para el desarrollo del trabajo se utilizaron métodos principalmente desde el enfoque cualitativo -observación directa y entrevista en profundidad- aplicados en los casos de estudio. A su vez, se realizó un rastreo bibliográfico que consistió en la consulta a fuentes de información secundaria para recolectar datos sobre la realidad histórica del sector y datos cuantitativos de censos de población.

Para conocer el total de asentamientos agrupados en cada caso se utilizaron imágenes satelitales disponibles en la plataforma Bingmaps, a partir de la cual se identificaron a través de interpretación visual, un poblado en cada distrito. Esta información se verificó mediante salidas a campo, donde a su vez se realizaron entrevistas en profundidad a la población local. Primero, se trabajó con los informantes claves reconocidos por la comunidad en cuanto a conocimientos sobre la historia del lugar y con los presidentes actuales y pasados de las comunidades Huarpes. Luego, se trabajó con aquellos habitantes que se localizan en los poblados y se entrevistaron también a pobladores que habitan de forma dispersa. Algunos habitantes realizan trabajos temporales en fincas ubicadas en las zonas irrigadas y por ello se trabajó con aquellos que se encontraban en los poblados al momento de realizar el trabajo de campo. Asimismo, entrevistamos a agentes estatales para indagar de qué manera el Estado -particularmente a escala municipal- percibe la configuración del hábitat rural y que políticas se desarrollan en el sitio.

Mediante la observación directa y la entrevista en profundidad se recabó información sobre el poblado, las actividades productivas que realiza la población, la disponibilidad de agua, la presencia del Estado en el sitio, el tipo de infraestructura y servicios disponibles y las percepciones de la población respecto a los cambios en el territorio y las ventajas/desventajas que implica habitar en el poblado a diferencia de los asentamientos dispersos. Después de la recolección de información, se construyeron categorías que guiaron el proceso de construcción de datos y su posterior análisis. Estas categorías se vinculan con la teoría expuesta en relación directa el trabajo de campo: Presencia de servicios e infraestructura, cantidad de viviendas, actividades productivas que se realizan en el poblado, accesibilidad a rutas, disponibilidad y formas de acceso al agua y políticas estatales aplicadas puntualmente en el sector de estudio y en las tierras secas no irrigadas en general.

El concepto de territorio como construcción social implica la apropiación material y simbólica de un espacio por parte de un individuo o grupos sociales. Milton Santos lo define como un espacio humano o espacio social, como un "conjunto de formas representativas de las relaciones sociales del pasado y del presente y por una estructura representada por las relaciones sociales que ocurren ante nuestros ojos y que se manifiestan por medio de los procesos y las funciones" (Santos, 1990: 138). En esta definición queda de relieve la importancia del abordaje diacrónico del territorio, ya que no se entiende como una realidad acabada, sino que se encuentra en permanente construcción (Raffestin, 2011). En esta línea, Sánchez (1991) postula a la consideración del tiempo y el espacio para su análisis y comprensión, desde la articulación de aspectos sociales, económicos y ecológicos que repercuten en su configuración. 
El territorio -con mayor énfasis en zonas rurales- muestra la interacción entre un grupo social determinado y la naturaleza, soslayando la dicotomía entre éstos, escenario que regía hasta mediados del siglo XX a diversas disciplinas y sesgando una parte de la realidad (Galafassi, 2001). Al respecto, Bifani (1999) expresa que "la naturaleza sufre siempre la acción transformadora del hombre y a su vez lo afecta y determina en un proceso dialéctico de acciones e interacciones" (1999: 31). En el proceso de construcción histórica del territorio se desprende la territorialización, entendida como "el intento por un individuo o grupo de afectar, influenciar y controlar personas, fenómenos y relaciones, por la delimitación y afirmación del control sobre un área geográfica" (Sack 1986: 26). La territorialidad resulta una construcción social dinámica y la mejor manera de estudiarlas es analizar su transformación y cambio en el tiempo (Marchionni et al., 2014: 19).

Mediante el abordaje histórico del territorio se obtienen datos para comprender las características del hábitat, donde a su vez, algunos autores abogan por la importancia de presentar atención al desenvolvimiento de la vida cotidiana pero en relación con diversas escalas de abordaje, tanto temporales como espaciales (Rapoport, 2003). El hábitat trata de una noción "más relacionante que estática de las expresiones, condiciones y realizaciones que surgen en la acción del habitar, de construir morada, de significar lugares" (Echeverría-Ramírez, 2003: 21). En el hábitat rural de tierras secas no irrigadas se encuentran elementos devenidos de la interacción naturaleza-cultura que se comprenden a partir de la articulación indivisible entre las actividades productivas y la vida familiar (Pastor, 2005).

\section{Lagunas del Rosario y La Asunción, entre la "abundancia de agua" y las acciones estatales}

El noreste de Mendoza se localiza dentro de la diagonal árida sudamericana de tierras secas. Se trata de una Amplia llanura árida afectada por fuertes procesos de desertificación (Abraham et al., 2014). Actualmente, el sector carece de escorrentía superficial de agua o sólo presenta desagües ocasionales (Abraham y Prieto, 1981). Pero a pesar de la sequía superficial, la llanura dispone en el subsuelo de un considerable reservorio de agua a poca profundidad que contribuye a mantener la vida en la zona (Abraham y Prieto, 1999). En el sitio predomina una economía de subsistencia donde la principal actividad es la cría de ganado caprino a escala familiar, aunque también se registran otras actividades como la venta de guano y la realización de artesanías. Además, la mayoría de los pobladores realizan migraciones temporales en época de cosecha para trabajar en las fincas cercanas.

Al interior de las tierras secas no irrigadas del noreste de Mendoza, la población se localiza de dos maneras: por un lado, de forma dispersa en torno a antiguos complejos lacustres, actualmente secos (Chiavazza y Prieto, 2004) y se accede a los mismos a través de huellas sobre el suelo arenoso que se desprenden de las rutas que cruzan el territorio. Por otro lado, de forma agrupada conformando pequeños poblados de entre 25 a 50 viviendas. En esta última forma de asentamiento se centra este trabajo. Tanto en La Asunción como en Lagunas del Rosario se ha encontrado un solo poblado en cada distrito. El poblado de Lagunas del Rosario (en adelante Lagunas) se encuentra a $100 \mathrm{~km}$ de distancia de la ciudad de Mendoza, mientras que el poblado de La Asunción (en adelante Asunción) se emplaza a $70 \mathrm{~km}$ de distancia. Lagunas se localiza en el interior del territorio y para poder acceder se realiza un desvío de $30 \mathrm{Km}$ a través de una vía de ripio que se 
desprende de la Ruta Nacional $n^{\circ} 40$. Asunción se encuentra al costado de la Ruta Nacional $n^{\circ} 142$ (Figura $\mathrm{N}^{\circ} 1$ ). La localización de cada poblado resulta clave en la compresión de los diferentes procesos que han incidido en su formación, donde la accesibilidad mediante la ruta incide en una mayor comunicación con los centros urbanos cercanos. Esto repercute de forma directa tanto en el desarrollo de las actividades productivas como en el abastecimiento de diversos bienes.

Figura $\mathrm{N}^{\circ} 1$

Localización de los casos de estudio en el departamento de Lavalle.

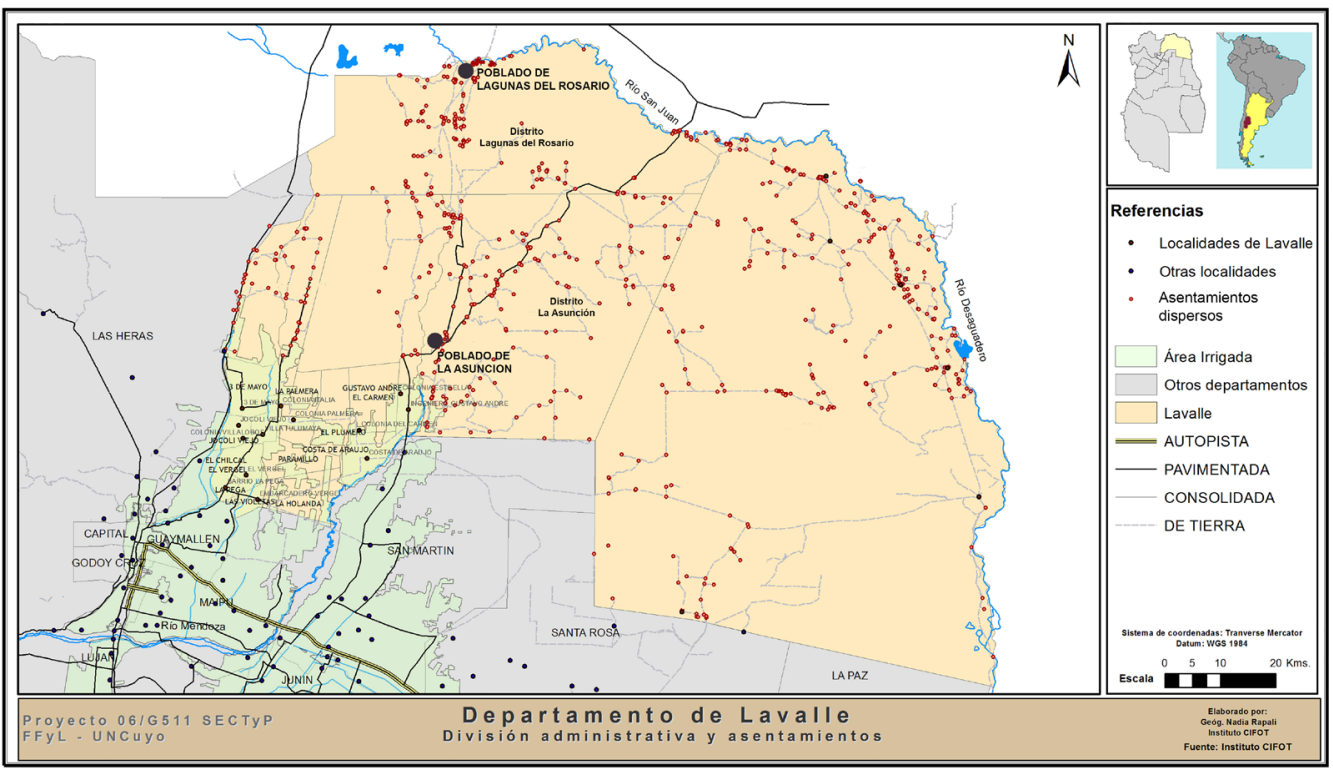

Fuente: Adaptación propia en base a Nadia Rapali, CIFOT, UNCuyo, 2012.

Respecto a la cantidad de habitantes, es inexistente el número de personas que habitan en estos poblados, ya que los datos estadísticos publicados por organismos oficiales abarcan a los distritos en su totalidad ${ }^{3}$ (considerando todo como población rural dispersa). Por esta razón, se realizó un relevamiento durante el trabajo de campo para estimar la población que reside de forma agrupada. Se registraron 35 viviendas en Lagunas y se ha considerado que habitan alrededor de 175 personas, calculando cinco usuarios por unidad habitacional. Para el caso de Asunción, se registraron 45 viviendas, lo que arroja una cantidad de 225 habitantes. En ambos casos, esta cantidad de viviendas se concentran en un radio aproximado de $350 \mathrm{~m}$.

El sector de estudio presenta transformaciones vinculadas, en un primer momento, con la merma en la disponibilidad de agua superficial y, en un segundo momento, con las acciones estatales en materia de infraestructura y servicios como respuesta a esos cambios ecológicos. Por ello, a continuación, se presenta un recorrido histórico con la finalidad de plasmar los aspectos más importantes que han incidido en las transformaciones del territorio y en la configuración del

Según el Instituto Nacional de Estadísticas y Censos (INDEC, 2010), la población total del distrito de Lagunas del Rosario es de 1042 habitantes y la población total del distrito de La Asunción es de 859 habitantes. 
hábitat agrupado. Luego, se exponen sus actuales características que se comprenden a la luz de esos cambios en el tiempo.

Históricamente, el noreste de Mendoza se caracterizó por la abundante presencia de agua superficial donde existían lagunas ${ }^{4}$ de considerable tamaño (Chiavazza, 2008). La mayor disponibilidad de agua favoreció el desarrollo de la pesca y la agricultura como actividades productivas principales hasta la primera mitad del siglo XX (Roig et al., 1999; Rusconi, 1961). Las formas de asentamiento en el sitio respondían en su totalidad a un patrón disperso, ribereño y costero (Abraham y Prieto, 1981). Esta organización se vinculaba con la óptima adaptación a las condicionantes ecológicas, donde "el modelo seleccionado para la mejor utilización de los recursos requería la dispersión; la agricultura no era posible en gran escala por la naturaleza del terreno" (Prieto, 1998: 61).

De esta época de "abundancia" hídrica, resaltan los edificios religiosos -capillas- como hitos históricos que dan cuenta de los procesos de territorialización en la época colonial. Al respecto Abraham y Prieto (1981) mencionan que

En la segunda mitad del siglo XVIII se establece la Junta de Poblaciones de Santiago de Chile que propicia la creación de centros urbanos en la zona de las lagunas, con el objeto de reunir a los indios dispersos. Este Intento de desestructurar el primitivo patrón de asentamiento, en la práctica nunca se llevó a cabo (...). De los pueblos planificados - Lagunas del Rosario, Asunción, San Miguel- sólo sus capillas se hicieron realidad (Abraham y Prieto, 1981: 130).

En el relato anterior queda de manifiesto las históricas intenciones de agrupar a los indígenas, aunque sin éxito. La principal causa de la dispersión radicaba en la comprensión de las características ecológicas del territorio por parte de este grupo social, para asegurar el control de una porción de tierra cultivable y disponibilidad de agua superficial.

De acuerdo a datos extraídos de las entrevistas y a diversos textos (Roig et al., 1999; Rusconi, 1961), el río Mendoza trasladó agua de manera constante hasta la década de 1940. Luego, se comienza a registrar la presencia estacional de agua en épocas estivales a causa del deshielo. La disminución del caudal se debió a su mayor uso y apropiación en la parte media de la cuenca del río Mendoza -donde se encuentra el oasis y la ciudad-por el desarrollo y éxito que alcanzó el cultivo de la vitivinicultura desde la primera mitad del siglo XX (Richard Jorba, 2000). El régimen de precipitaciones de la provincia oscila entre los 200 a $240 \mathrm{~mm}$ anuales y si se considera que el cultivo de la vid necesita entre 600 a $800 \mathrm{~mm}$ para su desarrollo, queda de manifiesto que la actividad vitivinícola es dependiente del agua proveniente de los ríos (Torres et al., 2003). A su vez, el crecimiento y desarrollo del oasis se apoyó en el uso de vegetación nativa, tanto para consumo de la población (leña y carbón) como para el desarrollo de la vitivinicultura (postes y rodrigones) y el tendido ferroviario (durmientes) (Abraham y Prieto, 1999). Por ello, se registra la extracción masiva de vegetación nativa en las zonas no irrigadas. La falta de agua superficial sumado a la tala del bosque nativo desencadenó procesos de desertificación en el noreste y este de Mendoza. Esto

La confluencia de los ríos Mendoza, San Juan y Desaguadero constituían el complejo hídrico denominado Lagunas de Guanacache (Lobos, 2004) 
afectó a las actividades productivas que históricamente se desarrollaban en el sitio -agricultura y pesca- y se propiciaron actividades de subsistencia centradas en la cría de ganado caprino, ya que esta actividad presenta mayor resistencia a las nuevas características ecológicas. La población recurrió al agua subterránea mediante la construcción de pozos aprovechando que la primera napa se encuentra a poca profundidad (entre $6 \mathrm{~m}$ a 10m). La década de 1970 se consigna como la época en que deja de llegar agua de forma definitiva hasta la parte baja de la cuenca debido al crecimiento sostenido del oasis.

Por los cambios ecológicos en el sector de estudio que impactaron en la economía de los pobladores y por ende en su calidad de vida, se registran una serie de acciones estatales con la finalidad de ayudar a la población local a alcanzar servicios básicos ${ }^{5}$. Ciertamente, en el periodo que abarca desde la década de 1940 a 1955 se advierte una serie de políticas públicas para extender los servicios sociales hacia las zonas rurales de la provincia (Hirschegger, 2010). En el departamento de Lavalle, se benefició tanto a zonas rurales irrigadas como a los territorios no irrigados, donde se proveyó de sala de primeros auxilios y escuelas primarias. También se sanciona la ley provincial n¹589 para la construcción de pistas de aterrizaje en zonas no irrigadas para el funcionamiento de "un equipo aéreo para el traslado de enfermos o accidentados, personal técnico y dotación de elementos sanitarios" (Hirschegger, 2010: 104). Estas acciones estatales se nuclearon hacia puntos de referencia tanto para el Estado como para la población. En el caso de Asunción y Lagunas fueron las capillas los edificios de referencia (Capilla Nuestra Señora del Rosario, en Lagunas y San Judas Tadeo de "El Cavadito" y Nuestra Señora de la Asunción, ubicada en el distrito de Asunción). Su elección no fue al azar, ya que una característica diferencial de la vida en el desierto nortino es la preponderancia de la figura de la iglesia católica, presente en el sitio desde la época colonial. Esta situación se refuerza en el hecho de que al Estado le ha resultado más alentador y factible la distribución de bienes y servicios hacia un sector determinado del territorio que hacerlo hasta diversos asentamientos dispersos.

En el año 1942 el Estado provincial construye el edificio de la escuela primaria en Lagunas del Rosario (La Quincena Social, 1942). Autoras locales como L. Katzer comenta que el poblado en torno a la capilla en Lagunas "se fue construyendo y consolidando (...) de la mano de la edificación de la escuela (...)" (Katzer, 2011: 44). No se han encontrado datos respecto al año de construcción del edificio escolar en Asunción, pero se infiere que la situación fue similar y que efectivamente estos servicios del Estado comenzaron a influir en el desarrollo de la configuración agrupada del hábitat, apoyado también en la baja disponibilidad de agua.

Entre los años 1947-1949 se construye en Lagunas del Rosario la sala de primeros auxilios (Hirschegger, 2010). Actualmente este edificio funciona como la vivienda del médico que trabajaba en la zona y se construyó una nueva sala a 400m de la capilla en la década de 1990, con comodidades que la antigua edificación no disponía. Además, los pobladores recuerdan en las entrevistas que el gobierno enviaba veterinarios hasta la zona de las capillas para asistir a los pobladores en el cuidado de las majadas.

El Estado moderno se interesa por el territorio de Lagunas y Asunción en el marco de la implantación del positivismo, que entiende que el progreso va de la mano del desarrollo y por lo tanto esa población y el territorio necesitaban de un giro transformador que sacara de "pobres" a sus habitantes (Lobos, 2004). 
Todos los entrevistados comentan que a partir de las acciones estatales desarrolladas a mediados del siglo XX se comienza a constatar la construcción de viviendas en torno a los edificios religiosos. Los pobladores de Lagunas del Rosario recuerdan que

"La gente que viene cree que siempre esto ha sido así, pero no. En la zona de la capilla sobre todo. Para hacer llegar agua hasta ahí eso era un desierto...Cuando queríamos hacer la Fiesta de la Virgen, que yo tenía unos 13 años, no se imagina como acarreábamos agua. No había ni una huella que sirviera. La gente pedía por el ferrocarril que trajeran tanques con agua y los almacenaban en los aljibes de la estación del ferrocarril y de ahí las llevábamos en carro hasta la capilla para que la gente tuviera agua durante la Fiesta. Ahora hay casas por todos lados, antes estaba la capilla sola y los puestos estaban alejados. Pregunte en qué año se empezó a poblar porque yo no me acuerdo...pero debe haber sido cuando dejó de venir agua. Y después se hizo la escuela y eso se fue poblando cada vez más" (Poblador 7, 60 años. Autor, Trabajo de campo, 2014).

Actualmente, en Lagunas el agrupamiento de viviendas se concentra en torno a la capilla (Declarada Monumento Histórico Nacional en 1975), donde se encuentra también la presencia de escuela primaria y secundaria, registro civil, museo, salón comunitario y centro de salud (Figura $\mathrm{N}^{\circ} 2$ ). Las viviendas se localizan al costado de un entramado de sendas, logrando la imagen de "poblado" que le imprime esta forma de organización en el territorio.

En Asunción, el patrón de asentamiento agrupado se emplaza alrededor de los servicios sociales como el nuevo edificio de iglesia católica (la iglesia vieja es un museo declarada patrimonio provincial), la escuela primaria, la posta sanitaria, una plaza central y un club deportivo (Figura $\mathrm{N}^{\circ} 3$ ). La organización comunitaria es un eje fundamental en el desarrollo habitacional y social del poblado (Autora, 2015).

Interesa destacar que el agua subterránea presenta altos niveles de salinidad (Grosso y Torres, 2015) y por ello los pobladores utilizan este recurso principalmente para el mantenimiento de las actividades pecuarias y en menor medida para consumo. Frente a esta situación, en el año 2007 el Estado municipal financiado por el Ente Nacional de Obras Hídricas y de Saneamiento comienzan las obras del denominado "acueducto del desierto", infraestructura que atraviesa el territorio del noreste de Mendoza con más de 270 km de recorrido para abastecer de agua a las comunidades Huarpes (Grosso y Torres, 2015). En la primera etapa, la red llegó hasta la localidad de El Cavadito (pasando por la comunidad de Asunción, poblado que se conectó posteriormente) y recién en el año 2010 llegó el servicio de agua al poblado de Lagunas. El acceso al agua desde el acueducto se realiza de forma comunitaria y para ello se dispone de una serie de grifos al costado de las sendas -distanciados entre sí cada $100 \mathrm{~m}$ a $200 \mathrm{~m}$ - donde los habitantes recurren al agua cuando se libera su paso, ya que no es un servicio constante. Por esta razón, se encuentran tanques de PVC de 1100 litros en cada vivienda para almacenar agua potable que el municipio traslada mediante camiones cisternas. Los poblados presentan buena accesibilidad desde las rutas principales, ya que el municipio en conjunto con la Dirección Provincial de Vialidad enripió el camino principal que atraviesa el territorio, facilitando la repartición de agua que se realiza aproximadamente cada 15 días a un mes en Lagunas y cada 15 días en Asunción. En los asentamientos dispersos se registra la repartición de agua cada 30 a 60 días. Por ello, la mejor accesibilidad al poblado incide de forma diferencial en la obtención de agua potable. 
Figura $\mathrm{N}^{\circ} 2$

Relevamiento del poblado de Lagunas del Rosario.
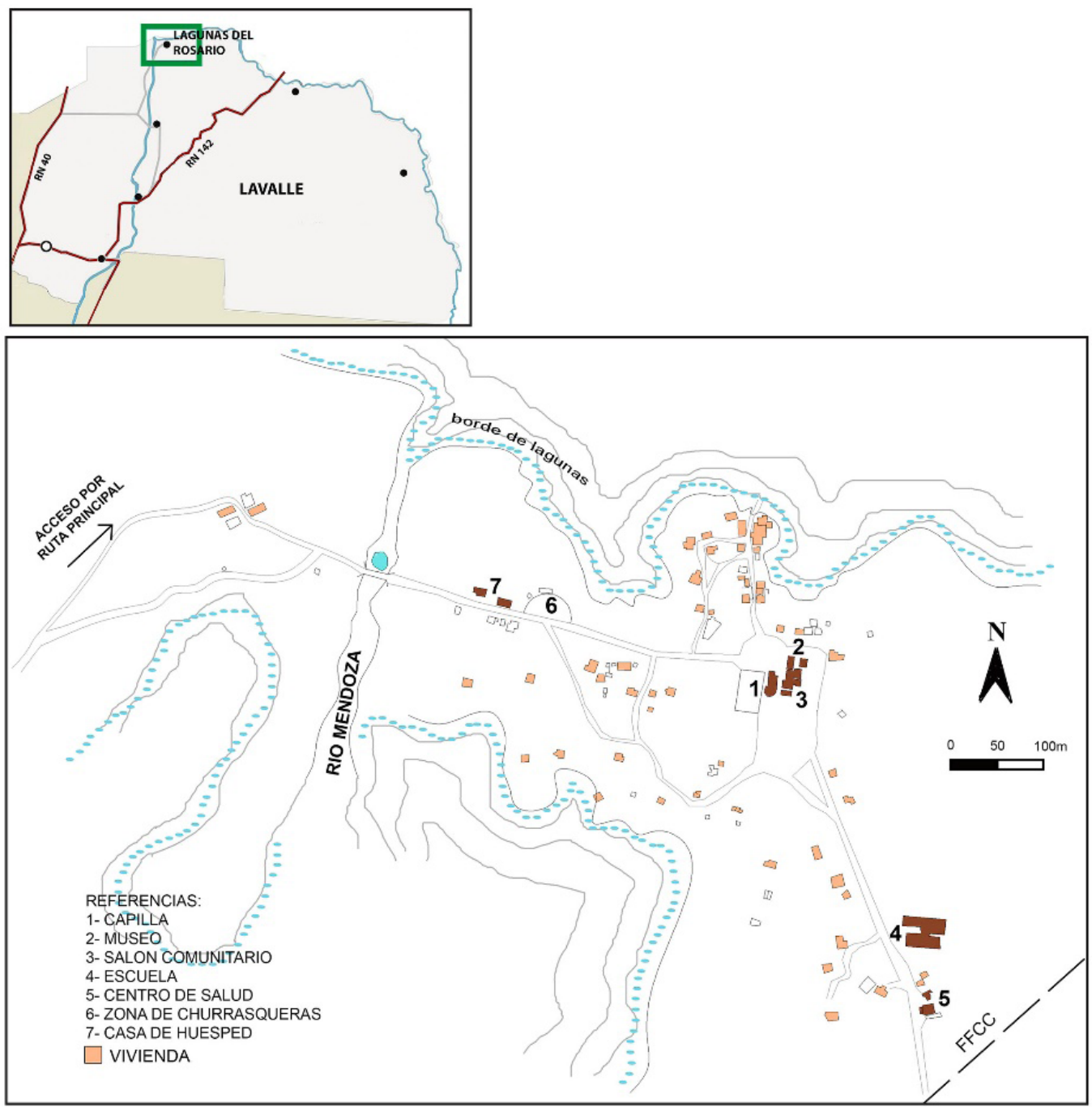

Fuente: Elaboración propia en base a Microsoft Bingmaps y trabajo de campo.

Otro de los servicios que cambió sustancialmente la cotidianeidad en los poblados fue la instalación del tendido de red eléctrica monofilar que en 1999 llegó hasta Lagunas del Rosario y en el año 2006 a Asunción. Los asentamientos dispersos, en cambio, cuentan en su mayoría con un panel fotovoltaico para el abastecimiento de energía eléctrica, desde el aprovechamiento de la radiación solar. Estos dispositivos se instalaron en el año 2008 y permitieron que los pobladores contaran con electricidad para el funcionamiento de dos focos y en algunos casos TV o radio. Es decir, resultó un abastecimiento limitado pero significativo para los pobladores. El problema se ha presentado varios años después cuando las baterías del sistema presentan baja capacidad de almacenamiento de energía sumado a que los pobladores no pueden asumir el costo que implica su reemplazo. Por ello, disponer de energía eléctrica permanente es otro de los motivos que ha impactado en el crecimiento del número de viviendas en los poblados. 
Figura $\mathrm{N}^{\circ} 3$

Relevamiento del poblado de Asunción.
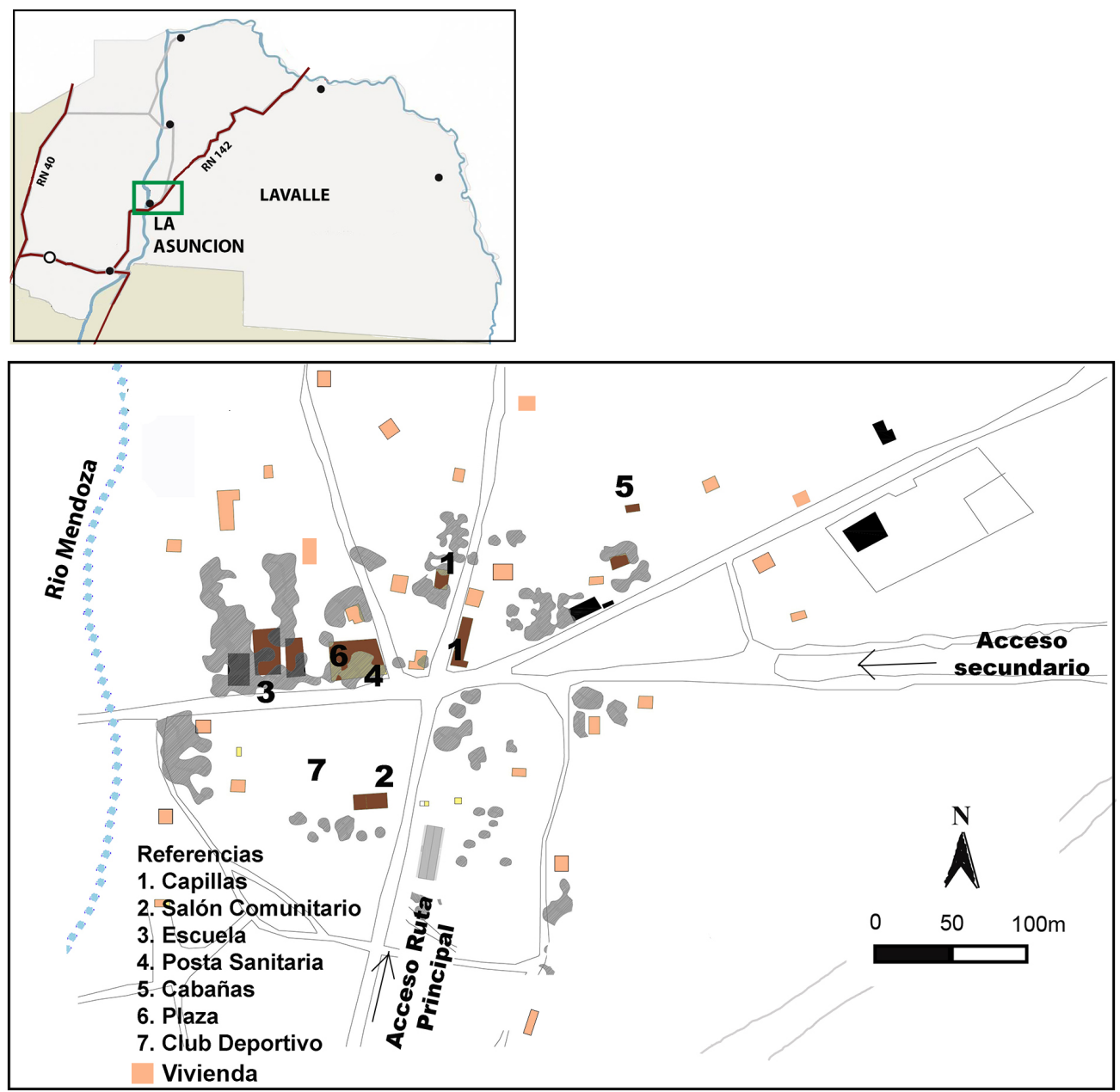

Fuente: Elaboración propia en base a Microsoft Bingmaps y trabajo de campo.

Respecto a las actividades productivas, la cría de cabras de forma extensiva ha sido la actividad principal en el noreste de Mendoza desde el desarrollo de los procesos de desertificación a mediados del siglo XX. Pero, la configuración del hábitat de forma agrupada incide de forma negativa en el desarrollo de esta actividad. En efecto, la mayor presencia de población en la misma superficie de terreno propició una mayor demanda de pasturas en los alrededores de los poblados, lo que se traduce en una mayor presión sobre el campo y en consecuencia la dificultad para mantener la actividad pecuaria. Por ello, actualmente es muy poca la población que se dedica a esta actividad en los poblados y quienes poseen majadas han construido los corrales a una distancia que oscila entre los $0,8 \mathrm{~km}$ a $2 \mathrm{~km}$ de distancia de la vivienda, para que las cabras estén en contacto con el campo y con mayor disponibilidad de pasturas. Esta situación implica la imposibilidad de control permanente de la majada y mayor vulnerabilidad frente a robos. A su vez, tanto en Lagunas como en Asunción, los corrales de los distintos pobladores están muy próximos entre sí -separados entre 400m a 900m de distancia- y por ende se propicia una competencia por 
las pasturas, impactando en el tamaño y desarrollo de la majada, donde actualmente se registran alrededor de 70 cabras por productor. En los asentamientos dispersos, la cantidad de cabezas de ganado ronda entre las 100 a 200 unidades.

Todos los entrevistados reconocen las dificultades que implica el desarrollo de la actividad pecuaria por el estado ecológico del campo y en relación directa con la cantidad de personas que habitan en el mismo sector. Desde los asentamientos localizados de forma dispersa advierten esta problemática y reconocen que el agrupamiento influye de forma negativa en el desarrollo de la actividad pecuaria.

“Pasa que el campo cerca de la capilla no sirve nada. Si no hay agua no está bueno el campo. Aparte que son muchos por aquella zona y el campo es menor para la cantidad de animales que tienen, en cambio acá tienen comida y agua" (Poblador 22, 56 años. Autor, Trabajo de campo, 2015).

Efectivamente, los pobladores cuyas actividades productivas se vinculan en mayor medida con la cría de ganado caprino se encuentran localizados de forma dispersa, manteniendo la tradicional forma de habitar el árido mendocino. Frente a las dificultades que presenta la cría de cabra en los poblados, otras actividades han adquirido protagonismo. Se encuentran empleos vinculados a los servicios sociales del Estado, la elaboración de artesanías en arcilla y madera, subsidios a la mitigación de la pobreza ${ }^{6}$, el auge del turismo rural y trabajos extraprediales centrados en la migración temporal a fincas cercanas. Estas actividades económicas sustentan la vida diaria de la mayoría de los habitantes de los poblados en relación con la pluriactividad, que se comprende "...como una estrategia que permite a los pequeños productores enfrentar los costes de su reproducción social en condiciones de creciente precariedad y pobreza" (Torres, 2008:214). Los empleos vinculados a los servicios del Estado son: maestros, limpiadores de escuelas, oficinas y enfermeros en el centro de salud, encargados de los museos, trabajos en relación con las tareas del registro civil y mantenimiento de las capillas. En relación con los trabajos extraprediales, los poblados cuentan con transporte público semanal que conectan con el centro urbano del departamento de Lavalle, lo que les permite disponer de una conexión directa que no poseen los asentamientos dispersos, ya que estos últimos tienen que trasladarse obligatoriamente hasta la ruta para alcanzar el servicio. Lo mismo sucede con la escuela: la posibilidad de contar con el centro educativo en el mismo poblado evita que los alumnos se trasladen varios kilómetros diarios para alcanzar el servicio.

También resalta el incipiente, aunque creciente turismo. Esta actividad se ha convertido en un motor importante para el desarrollo económico de los poblados, que intenta poner en valor a la cultura Huarpe y a las actividades y productos que se ofrecen (De Simón, 2013) abogando por sus características socio-históricas. Asunción posee mayor experiencia y actualmente se encuentra en potencial desarrollo, donde destacan tres circuitos principales: turismo rural, cultural-religioso y naturaleza-aventura. Desde el año 2007, existe un proyecto de Turismo Comunitario denominado Tintihuili Kanay Ken (que significa en vocablo Huarpe "ramblón de amigos") impulsado por familias de la comunidad con el acompañamiento del Instituto Nacional de Tecnología Agropecuaria (INTA), a través del Programa de Cambio Rural y con el apoyo de la Dirección de Turismo y

Puntualmente Asignación Universal por Hijo y Plan Jefes y Jefas de Hogar. 
Cultura del Municipio de Lavalle. La actividad turística en Asunción es fuertemente promocionada por la Administración Pública local, invirtiendo presupuesto, recurso humano y maquinarias a disposición de las familias que susciten esta actividad con estrecha relación político-institucional. Una particularidad de la actividad turística de Asunción es el desarrollo gastronómico, que genera nuevas alternativas laborales y que involucran a todo el núcleo familiar.

"Además de preparar comida típica, la idea es que los turistas compartan y conozcan a las familias del lugar y que vean varios productos como telares, cueros..." (Entrevista realizada a familia, Autora, Trabajo de campo, 2013).

Lagunas, en cambio, presenta un bajo desarrollo turístico pero los habitantes han tomado como referente a la comunidad de Asunción y el desarrollo que ha tenido esa actividad con la finalidad de organizarse junto con el municipio para plantear un nuevo producto adaptado a la realidad en tierras secas no irrigadas.

"Ahora vinieron del municipio a capacitarnos en historia y marketing porque quieren que empiecen a llegar turistas a la capilla y que nosotros los atendamos y les vendamos cosas. Yo pedí que dieran el curso de marketing porque acá no sabemos cómo vender los productos. Son productos muy lindos los que hacemos pasa que la mayoría son grandes y la gente por ahí viene y quiere llevarse un recuerdo chiquito, como un imán y esas cosas chiquitas no estamos acostumbrados a hacer" (Pobladora 8, 65 años. Autor, trabajo de campo, 2015).

La actividad turística en ambos poblados es viable por su buena accesibilidad y por la presencia de infraestructura y servicios. A pesar de que deberían realizarse mejoras para adecuarlos frente a los requerimientos turísticos, estos sitios cuentan con churrasqueras, espacios de sombra, juegos infantiles, centro de salud y red de agua. El auge turístico se constata desde las fiestas religiosas que se celebran en los poblados y que cada año adquiere mayor relevancia e incorpora más adeptos.

Interesa destacar también que mediante el agrupamiento de viviendas se facilitó la organización comunitaria y la "visibilización" de la población residente en el sector de estudio hacia el Estado municipal en primer lugar y posteriormente en una escala provincial. En efecto, la organización social y el agrupamiento habitacional fueron centrales para el reclamo de las garantías mínimas de habitabilidad.

De acuerdo a lo expuesto, la configuración del hábitat de forma agrupada fue adquiriendo protagonismo a partir de mediados del siglo XX desde dos situaciones: la primera se vincula con la merma en la disponibilidad de agua superficial y con el desarrollo de procesos de desertificación, que incidió en las actividades productivas que históricamente se desarrollaban en el sitio -agricultura y pesca- y se favorece una economía de subsistencia centrada en la cría de cabra. La segunda situación se vincula con la presencia del Estado desde la provisión de infraestructura y servicios que primero implicó la construcción de escuelas y centros de salud y que en las últimas décadas involucraron a los servicios de agua, electricidad y mejor accesibilidad con los poblados urbanos próximos. Además, se observa la posibilidad de contar con empleos en relación con los servicios del Estado y el turismo, que se trata de sueldos fijos que no dependen del éxito en la 
producción pecuaria, que se ve dificultada por la cantidad de personas viviendo en un mismo sector del territorio. Por ello, los pobladores que se dedican a esta actividad se localizan de forma dispersa para asegurar disponibilidad de pasturas.

En tierras secas no irrigadas del noreste de Mendoza se encuentra un patrón de asentamiento agrupado, que tiene que ver con las transformaciones del territorio y las adaptaciones de algunos pobladores. Desde aquí nos planteamos trasladar estos resultados a la planificación de políticas públicas: ¿Las transformaciones habitacionales y productivas de las zonas rurales no irrigadas son consideradas en el diseño-implementación de las políticas públicas? ¿Cómo observa el Estado la presencia de estos poblados como contracara de los asentamientos dispersos?

Las intervenciones del Estado en el sector de estudio favorecen la concentración de la población en zonas cercanas a los servicios sociales. A partir de las entrevistas se constata que la mayor parte de las acciones tienden a implementarse en los poblados y que aquellos habitantes dispersos que quieren acceder a los beneficios deben trasladarse hasta el sitio. Es decir que continúan siendo las capillas los puntos de referencia hacia donde se canalizan en mayor medida las acciones estatales En efecto, al concentrar las acciones estatales en sectores determinados $Y$ se relega a un segundo plano a la población que se localiza de forma dispersa y que se caracteriza por la cría extensiva de ganado caprino. A su vez, considerar que en los poblados la gente también se dedica exclusivamente a la cría de cabras sin considerar que priman otras actividades impide la formulación de políticas acordes a la realidad económica de los habitantes. En este sentido, se observa cómo se generan tensiones territoriales vinculadas a la falta de reconocimiento de las diversas formas del hábitat rural, que no sólo implica a la vivienda sino que también involucra a las actividades productivas que se realizan y los servicios utilizados en la cotidianeidad, como la escuela, acceso al agua y a la electricidad. Por ello, se disiente con los textos que exponen que en las tierras secas no irrigadas de Mendoza se encuentran exclusivamente poblaciones dispersas dedicadas a la cría de ganado caprino y se aboga por la consideración del hábitat agrupado y disperso como dos formas de habitar las tierras secas no irrigadas.

\section{Conclusiones}

La presencia del Estado y sus intervenciones con la dotación de servicios e infraestructura, sumado a los procesos de desertificación y falta de agua superficial, establecieron las bases para la trasformación paulatina del patrón tradicional disperso hacia un patrón agrupado. La existencia de los asentamientos agrupados implica la revisión en las políticas que el Estado lleva adelante en el sector, ya que no se trata de actividades económicas vinculadas exclusivamente con la cría de ganado caprino, sino que aparecen nuevas actividades que derivan de la combinación de factores ecológicos, sociales y económicos. Estas características deberían contemplarse en las actuales políticas hacia el territorio local con la finalidad de asegurar la mejor intervención y que resulten coherentes con las características naturales y culturales de inserción.

La heterogeneidad en la construcción del hábitat se presenta como un hallazgo para desalentar las normativas que tiendan a la consideración racional e instrumental del territorio y por ende a la homogeneización de su población. Ciertamente, a partir del estudio de las realidades actuales se pretende poner en valor a la diversidad como herramienta para la planificación del territorio. 
La consideración de la temporalidad en el estudio del hábitat rural presenta potencialidades para comprender y analizar las transformaciones del territorio y las estrategias que despliegan los pobladores para adaptarse a esos cambios. En este trabajo se ha tratado de poner en tensión lo tradicional con lo emergente, es decir, que sin desconocer la "tradición" de lo disperso, se ha buscado comprender las nuevas formas de asentamiento agrupado, sus características y los factores naturales y antrópicos que inciden en su desarrollo, con vistas a un ordenamiento territorial más inclusivo.

\section{Bibliografía}

ABRAHAM, E. y PRIETO, R. Enfoque diacrónico de los cambios ecológicos y de las adaptaciones humanas en el noreste árido mendocino. Cuadernos del CEIFAR, 1981, Vol. 8, p.109-139.

ABRAHAM, E. y PRIETO, R. Guanacache, la travesía de los profundos cambios. En: GOTTHELF, R. Guanacache, Fidel Roig Matons, pintor del desierto. Mendoza: EDIUNC, 1999, p. 107-125.

ABRAHAM, E.; SORIA, D.; RUBIO, C.; RUBIO, M.; VIRGILLITO, J. Síntesis diagnóstica del modelo de organización territorial. Mendoza, Argentina. Subsistema físico-biológico o natural de la provincia de Mendoza. Documento técnico. Mendoza: UNCUYO, 2014.

BIFANI, P. Medio ambiente y desarrollo sostenible. Madrid: IEPALA, 1999.

CASTRO, H. y REBORATTI, C. Revisión del concepto de ruralidad en la Argentina y alternativas posibles para su redefinición. Buenos Aires: Secretaría de Agricultura, Ganadería, Pesca y Alimentos, 2008.

CHIAVAZZA, H. Cambios ambientales y sistemas de asentamiento en el árido normendocino. Arqueología en los paleocauces del Río Mendoza (Tesis doctoral). Buenos Aires: Universidad Nacional de La Plata, 2008.

CHIAVAZZA, H. y PRIETO, C. Arqueología, ambiente y cultura en el noreste de Mendoza. XV Congreso Nacional de Arqueología Argentina, 2004. Disponible en internet: http://bdigital.uncu.edu. $\operatorname{ar} / 2702$

DE SIMON, C. Planificación turística sustentable en el departamento de Lavalle, Mendoza, Argentina. TURyDES, 2013, Vol. 6, № 15. Disponible en internet: http://www.eumed.net/rev/turydes/15/ turismo-sustentable-mendoza.pdf

ECHEVERRIA RAMIREZ, C. Hábitat versus vivienda. Mirada crítica sobre el viviendismo. En: Actas del Seminario-Foro Internacional La construcción del hábitat popular: Experiencias de intervenciones urbanas, arquitectónicas, tecnológicas y pedagógicas. Bogotá: Universidad La Gran Colombia, 2003.

GALAFASSI, G. Las preocupaciones por la relación naturaleza-sociedad. Ideas y teorías en los siglos XIX y XX. Una primera aproximación. Theomai, 2001, Nº 3. Disponible en internet: http://www. redalyc.org/pdf/124/12400311.pdf 
GROSO, V. y TORRES, L. Entre las políticas por el agua y los esfuerzos por calmar la sed. El "acueducto del desierto" en las tierras secas no irrigadas de Lavalle, Mendoza. América Latina Hoy, 2015, Vol., 69, p. 17-33.

GUDIÑO, E. Ordenar el territorio: Un desafío para Mendoza. Mendoza: EDIUNC, 2017.

HIRSCHEGGER, I. Alcance territorial de las políticas públicas peronistas en la Provincia de Mendoza (Argentina). 1946-1955. Apuntes, 2010, № 66, p. 83-115.

INSTITUTO NACIONAL DE ESTADÍSTICAS Y CENSOS (INDEC). Censo Nacional de Población, Hogares y vivienda, Buenos Aires: INDEC, 2010.

KATZER, L. Praxis indígena y gubernamentabilidad. Una etnografía de los procesos de producción territorial Huarpe en la provincia de Mendoza (Tesis doctoral). Buenos Aires: Universidad Nacional de La Plata, 2011.

LA QUINCENA SOCIAL. De Laguna del Rosario, La quincena social, n559-560, 1942, s/p.

LOBOS, N. Para pensar la identidad cultural en el desierto de Lavalle Revista Confluencia, 2004, N4, p. 1-20.

MARCHIONNI, F; MORENO, M.; D'AMICO, P.; ACCORINTI, C.; ESTEVES, M.; SALES, R.; TORRES, L.; PASTOR, G.; ZALAZAR, M. y PESSOLANO, D. Territorio y tierras secas: reflexiones teóricas desde miradas interdisciplinarias. En: TORRES, L.; ABRAHAM, E. y PASTOR, G. Territorio y Tierras secas: Reflexiones teóricas desde miradas interdisciplinarias Ventanas sobre el territorio. Herramientas teóricas para comprender las tierras secas, Mendoza: EDIUNC, 2014, p.11-29.

MINISTERIO DEL INTERIOR. Directrices para el ordenamiento territorial de las áreas rurales de Mendoza. Informe integrado. 2014. Disponible en internet: https://www.mininterior.gov.ar/planificacion/pdf/planes-prov/MENDOZA/Directrices-para-el-Ordenamiento-Territorial-de-las-areas-rurales-de-Mendoza.pdf

MONTAÑA, E. Escenarios de cambio ambiental global, escenarios de pobreza rural. Buenos Aires: CLACSO, 2013.

MONTAÑA, E. Identidad regional y construcción del territorio en Mendoza (Argentina): memorias y olvidos estratégicos. Bulletin de l'Institut français d'études andines, 2007, Vol. 36, №2, p. 277-297.

PASTOR, G. Patrimonio, vivienda y agua en el paisaje del noreste mendocino. En: FERNÁNDEZ, A. y ABRAHAM, E. El agua en Iberoamérica. Uso y gestión del agua en tierras secas. Mendoza: CYTED, 2005, p. 79-92.

PRIETO, R. Formación y consolidación de una sociedad en un área marginal del Reino de Chile: La provincia de Cuyo en el Siglo XVII. Anales de Arqueología y Etnología, 1998, № 52-53, p. 17-366.

QUIROGA MARTINEZ, R. Indicadores ambientales y de desarrollo sostenible: Avances y perspectivas para América Latina y del Caribe. Santiago de Chile: CEPAL, 2007. 
RAFFESTIN, C. Por una geografía del poder. Traducción por VILLAGÓMEZ VELÁZQUEZ, Y. México: El Colegio de Michoacán, 2011.

RAPOPORT, A. Cultura, arquitectura y diseño. Barcelona: Edicions UPC, 2003.

RICHARD JORBA, R. Modelos vitivinícolas en Mendoza (Argentina). Desarrollo y transformaciones en un período secular, 1870-2000. Historia Económica \& Historia de Empresas, 2000, Vol.3, №1, p.111-148.

ROIG, F.; ROIG, A.; ROIG, M.; ROIG, V. \& ROIG, E. Guanacache. Fidel Roig Matóns, pintor del desierto. Mendoza: EDIUNC, 1999.

RUSCONI, C. Poblaciones pre y post hispánicas de Mendoza. Volumen I: Etnografía. Mendoza: Imprenta Oficial, 1961.

SACK, R., La territorialidad humana. Su teoría y la historia. Nueva York: Cambridge University Press, 1986.

SANCHEZ QUINTANAR, C. y JIMENEZ ROJAS, E. La vivienda rural. Su complejidad y estudio desde diversas disciplinas. Luna Azul, 2010, N³0, p. 174-196.

SANCHEZ, E. Espacio, economía y sociedad. España: Siglo XXI Editores S.A., 1991.

SANTOS, M. Por una geografía nueva. Madrid: Espasa Calpe, 1990.

SECRETARIA DE AMBIENTE Y ORDENAMIENTO TERRITORIAL. Plan Provincial de Ordenamiento Territorial. Mendoza: Gobierno de Mendoza, 2017

TORRES, L. Nueva ruralidad en territorios periféricos: los productores caprinos del noreste de Mendoza (Argentina). Universitas humanística, 2008, № 66, p. 199-218.

TORRES, L.; ABRAHAM, E.; TORRES, E. y MONTAÑA, E. Acceso a los recursos y distribución de la población en tierras secas de Argentina: el caso de Mendoza. Aportes hacia la equidad territorial. Scripta Nova, 2003, Vol. VII, N¹48. Disponible en internet: http://www.ub.edu/geocrit/sn/sn-148. htm 